\title{
Breast cancer in pregnancy; routine examination, diagnosis and management: A case report
}

\author{
Anne-Beatrice Kihara ${ }^{1,2^{*}}$, Kiarie Gladwell ${ }^{1,2}$, Cheserem J. Eunice ${ }^{1,2}$, Amin Medhat $^{1,2}$, \\ Bashir Issak ${ }^{3}$, Rogena Emily, ${ }^{2,4}$ Kosgei J. Rose ${ }^{1,2}$ \\ ${ }^{1}$ Department of Obstetrics and Gynaecology, University of Nairobi, Nairobi, Kenya; \\ *Corresponding Author: ruby medical@yahoo.com \\ ${ }^{2}$ Department of Reproductive Health, Kenyatta National Hospital, Nairobi, Kenya \\ ${ }^{3}$ Division of Reproductive Health, Ministry of Public Health and Sanitation, Kenya \\ ${ }^{4}$ Department of Human Pathology, University of Nairobi, Nairobi, Kenya
}

Received 20 December 2012; revised 1 April 2013; accepted 16 April 2013

Copyright (C) 2013 Anne-Beatrice Kihara et al. This is an open access article distributed under the Creative Commons Attribution License, which permits unrestricted use, distribution, and reproduction in any medium, provided the original work is properly cited.

\begin{abstract}
Pregnancy associated breast cancer is defined as breast cancer diagnosed during pregnancy or in the first postpartum year. The incidence is rare; however it is important to be aware of its occurrence with the increased challenges posed by delays in diagnosis, treatment and optimization of maternal and fetal outcomes. The case report presented is of a 36 years old, Para $0+2$ known diabetic and hypertensive pregnant woman, first seen in the antenatal clinic at 14 weeks of gestation. The patient requested for a breast examination following National campaigns conducted during the International Breast Cancer Awareness month. The examination revealed a grossly enlarged right breast with "peau de orange" skin changes. The patient eventually underwent radical mastectomy. Histopathology revealed a grade 3 invasive ductal carcinoma with lymphovascular invasion (Stage TNM). This case is presented to highlight missed opportunities in antenatal clinic settings for screening, diagnosis and treatment of cancer of the breast in pregnancy. The role of a multidisciplinary approach to management has also been emphasized.
\end{abstract}

Keywords: Pregnancy; Breast Cancer; Screening; Multidisciplinary Management

\section{INTRODUCTION}

Women are increasingly postponing pregnancy and child birth to an older age increasing their risk for on- cological malignancies associated with pregnancy including, breast cancer. Pregnancy associated breast cancer affects approximately 1:3000 with an average age of 32 - 38 years. In $6.5 \%$ of all cases, it affects women less than 40 years [1]. Anatomical and physiological changes that occur in the breast during pregnancy mimic symptoms of cancer, posing challenges in diagnosis. When cancer of the breast diagnosis is made during pregnancy, the clinician is faced with a dilemma in treatment that optimizes obstetric outcomes and at the same time does not worsen the disease prognosis. Striking this balance is difficult, because the mother and baby are intricately intertwined for survival and worse when a Primigravida or a patient with bad obstetric history such as the case presented.

\section{CASE REPORT}

A 36 years old, Para $0+2$ known diabetic and hypertensive pregnant woman, first presented to the antenatal clinic at 14 weeks of gestation. The previous pregnancy losses were in the early second trimester. Her antenatal care profile was normal, she was normotensive and her random blood sugar was normal. Her clinic visit coincided with the International Breast Cancer Awareness month and the patient asked for a breast examination having seen flyers in the clinic reception.

Physical examination of the breast revealed a grossly enlarged right breast with "peau de orange" skin (Figure A1). There was no tenderness or palpable mass or palpable lymph nodes. The nipple was grossly normal. The fundal height was 14 weeks and corresponded to the gestational age. All other system examinations were normal. An obstetric scan showed a normal viable fetus at 14 weeks of gestation and no evidence of cervical in- 
competence.

Breast ultrasound revealed a hypo echoic mass in the right breast. Fine needle aspiration and cytology revealed evidence of malignant ductal cells. At this point the patients' greatest concern was her unborn baby given the bad obstetric history.

After consultation with a multidisciplinary team comprising of the Obstetrician, Surgeon, Neonatologist and Medical Therapeutic Oncologist; a decision to have the patient undergo right radical mastectomy was made. After counseling, the patient consented to the surgery.

Post operatively the histopathology was reported as grade 3 invasive ductal carcinoma of the breast with all 8 lymph nodes involvement (not otherwise specified (NOS) pattern). There was lymphovascular invasion and intense lymphocytic infiltration. The imunohistochemistry revealed estrogen receptor negative, progesterone receptor equivocal and HER 2 positive (Figures A2 and A3).

Following the mastectomy the patient was counseled to commence chemotherapy (Cyclophosphamide, Adriamycin and 5 Florouracil) at 22 weeks of gestation, which she consented for. She received two courses of chemotherapy with one month interval between the courses. After her second course the patient went into premature labor at 27 weeks of gestation and had spontaneous vaginal delivery to a live baby whose Apgar score was 8 in one minute and 10 in five minutes, and weighed 1050 grams. The baby was admitted to neonatal intensive care unit under the care of the Neonatologist. The mother is continuing cancer treatment with the Oncologist and postnatal care with the Obstetrician.

\section{DISCUSSION}

Breast examination must be included as a routine physical examination during each antenatal visits for all pregnant women. Presence of a breast mass, pathological nipple discharge and skin changes increases the index of suspicion of cancer of the breast. Mastalgia is usually not an indication of underlying malignancy [2,3]. Breast ultrasound should be carried out for any suspicious breast mass or when there is a high index of suspicion for breast cancer [4]. Mammogram is contradicted in pregnancy [5]. A confirmed mass should be biopsied for histology or fine needle aspiration for cytology done. Breast cancer staging is possible in pregnancy and prognostic factors like presence of oestrogen and progesterone receptors, HER2 status, BRCA testing can be done. Pregnancy poses a diagnostic challenge for breast cancer due to the anatomical and physiological variation represented by lactation changes [6] such as increased nuclear cytoplasmic ration and the presence of nucleoli. These anatomical and physiological changes mimic signs of breast cancer hence the need to shift from opportunistic breast examinations in pregnancy, to routine health provider based breast examinations. In the case presented, the breast examination was an opportunistic examination through the client's request, being the International Breast Cancer Awareness month. Were it not for the awareness month, the diagnosis of grade 3 invasive ductal carcinoma of the breast may have been missed.

There is poorer prognosis for patients diagnosed with advanced disease in pregnancy and in the post partum period [7].

Once a diagnosis of breast cancer in pregnancy is made, a multidisciplinary team must be constituted; this has been shown to optimize patient care [8-10]. Various treatment modalities should be discussed with the patient and the family. The stage of breast cancer, gestational age and obstetric history must be put to context during the discussion with maternal health always taking precedence. Abortion is not routinely recommended. However, in cases of advanced metastatic disease during the first 14 weeks of pregnancy; chemotherapy promptly needs to be instituted and with estrogen receptor positive cells termination of pregnancy maybe proposed [11,12]. Surgery is possible in all trimesters, however in first trimester modified radical mastectomy is recommended. Radiotherapy is possible in pregnancy but dependent on fetal dose received as it can result in poor fetal outcomes hence need to individualize cases. Neo-adjuvant or adjuvant dose dense chemotherapy started from 14 weeks of gestation has been shown to reduce teratogenicity and premature delivery. Methotrexate, Tamoxifen and Trastuzmab are contraindicated in pregnancy $[13,14]$. In the case presented she received chemotherapy at 22 weeks of gestation and after two courses went into premature delivery at 27 weeks of gestation.

When premature delivery does not occur, the timing of delivery should be close to term. The chemotherapy should be stopped at 36 weeks of gestation to avoid maternal and fetal leucopenia. The route of delivery should be vaginal, caesarian section is reserved for the usual obstetric indications.

Breast feeding from the treated breast is not contraindicated. However, reduced milk volume due to scar tissue, limits ability to breastfeed. It is contraindicated to breastfeed while the mother is under chemotherapy or radiotherapy. Recurrence and survival of previously treated breast cancer does not appear to be adversely affected by a subsequent pregnancy especially in patients who have been in remission over five years $[15,16]$.

In conclusion, breast examination must be made routine in antenatal care clinics. In cases where there is a high index of suspicion for breast cancer, screening must be done and diagnosis confirmed from tissue histopathology. Once a diagnosis of cancer of the breast in pregnancy is made a multidisciplinary team should be constituted and the patient counseled on available treat- 
ment options. In all stages of management, the life of the mother should take precedence and she should be an active participant in her management. We recommend hypothesis driven research to determine the best screening, diagnostic and treatment approaches of cancer of the breast in pregnancy.

\section{REFERENCES}

[1] Keyser, E.A., Staat, B.C., Fausett, M.B. and Shields, A.D. (2012) Pregnancy-associated breast cancer. Reviews in Obstetrics and Gynecology, 5, 94-99.

[2] Salzman, B., Fleegle, S. and Tully, A.S. (2012) Common breast problems. American Family Physician, 86, 343349.

[3] Woo, J.C., Yu, T. and Hurd, T.C. (2003) Breast cancer in pregnancy: A literature review. Archives of Surgery, 138, 91-98.

[4] Theriault, R. and Hahn, K. (2007) Management of breast cancer in pregnancy. Current Oncology Reports, 9, 17-21. doi:10.1007/BF02951421

[5] Liberman, L., Giess, C.S., Dershaw, D.D., Deutch, B.M. and Petrek, J.A. (1994) Imaging of pregnancy-associated breast cancer. Radiology, 191, 245-248.

[6] Hassiotou, F. and Geddes, D. (2012) Anatomy of the human mammary gland: Current status of knowledge. Clinical Anatomy, 26, 29-48. doi:10.1002/ca.22165

[7] Azim Jr., H.A., Santoro, L., Russell-Edu, W., Pentheroudakis, G., Pavlidis, N. and Peccatori, F.A. (2012) Prognosis of pregnancy-associated breast cancer: A meta-analysis of 30 studies. Cancer Treatment Reviews, 38, 834-842. doi:10.1016/j.ctrv.2012.06.004

[8] Zagouri, F., Sergentanis, T.N., Chrysikos, D., Dimitrakakis, C., Tsigginou, A., Zografos, C.G., Dimopoulos, M.A. and Papadimitriou, C.A. (2012) Taxanes for breast cancer during pregnancy: A systematic review. Clinical

\section{Breast Cancer.}

[9] Cordoba, O., Llurba, E., Saura, C., Rubio, I., Ferrer, Q., Cortes, J. and Xercavins, J. (2012) Multidisciplinary approach to breast cancer diagnosed during pregnancy: Maternal and neonatal outcomes. Breast. doi:10.1016/j.breast.2012.10.005

[10] Brewer, M., Kueck, A. and Runowicz, C.D. (2011) Chemotherapy in pregnancy. Clinical Obstetrics and Gynecology, 54, 602-618. doi:10.1097/GRF.0b013e318236e9f9

[11] Lenhard, M.S., Bauerfeind, I. and Untch, M. (2008) Breast cancer and pregnancy: Challenges of chemotherapy. Critical Reviews in Oncology/Hematology, 67, 196203. doi:10.1016/j.critrevonc.2008.02.007

[12] Daniilidis, A., Giannoulis, C., Sardeli, C., Dinas, K., Nasioutziki, M., Tantanasis, T., Loufopoulos, A. and Tzafettas, J. (2010) Pregnancy-associated breast cancer-A review analysis. European Journal of Gynaecological Oncology, 31, 485-490.

[13] Amant, F., Loibl, S., Neven, P. and Van Calsteren, K. (2012) Breast cancer in pregnancy. Lancet, 379, 570-579. doi:10.1016/S0140-6736(11)61092-1

[14] Cardonick, E., Gilmandyar, D. and Somer, R.A. (2012) Maternal and neonatal outcomes of dose-dense chemotherapy for breast cancer in pregnancy. Obstetrics \& Gynecology, 120, 1267-1272.

[15] Keleher, A.J., Theriault, R.L., Gwyn, K.M., Hunt, K.K., Stelling, C.B., Singletary, S.E., Ames, F.C., Buchholz, T.A., Sahin, A.A. and Kuerer, H.M. (2002) Multidisciplinary management of breast cancer concurrent with pregnancy. Journal of the American College of Surgeons, 194, 54-64. doi:10.1016/S1072-7515(01)01105-X

[16] Slingerland, M., Kroep, J., Liefers, G.J., Scholten, A. and Nortier, J.W. (2012) Pregnancy-associated breast cancer: Current opinions on diagnosis and treatment. Nederlands Tijdschrift voor Geneeskunde, 156, A5286. 


\section{APPENDIX 1}

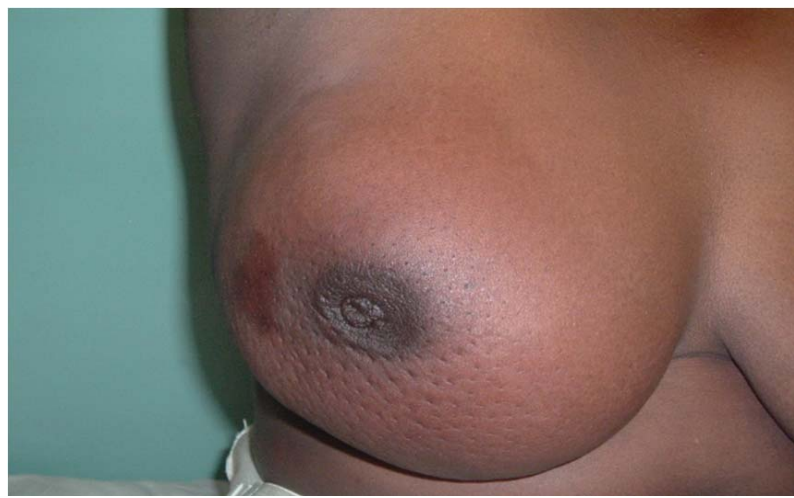

Figure A1. Enlarged right breast with peau de orange skin and nipple retraction.

\section{APPENDIX 2}

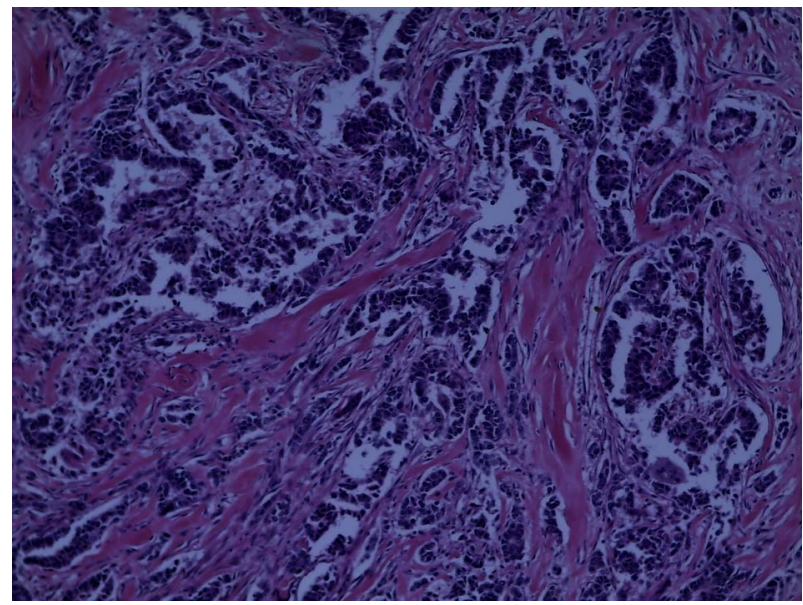

Figure A2. H/E stain, $\times 10$ magnification, sections showing invasive ductal carcinoma (NOS pattern).

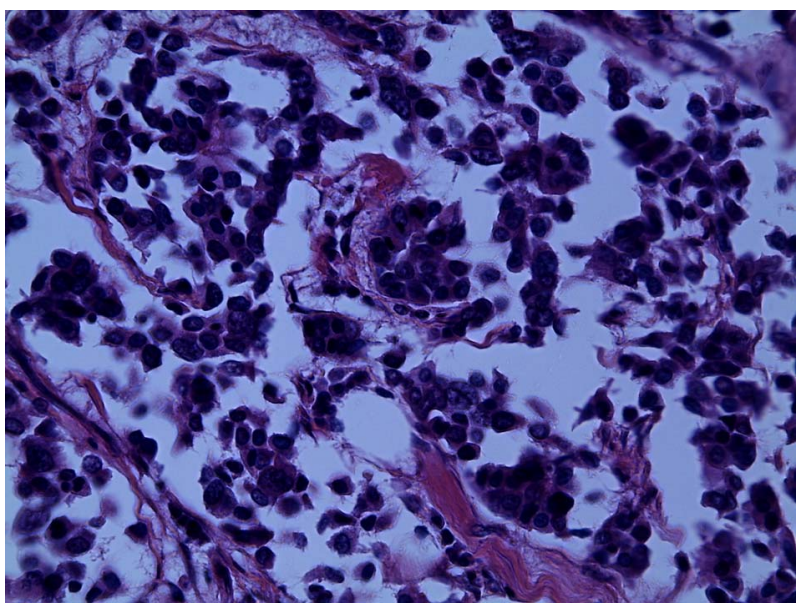

Figure A3. H/E stain, $\times 40$ magnification; sections showing shrinkage due to fixation artefacts as a result of poor fixation, marked pleomorphism and poorly formed glandular structures and clusters. 